\title{
Integration of materials visualization with a materials database in a Materials Science and Engineering freshman course
}

$\begin{array}{cccc}\text { Kisung Kang } & \text { Matthew D Goodman } & \text { Jessica A Krogstad } & \text { Cecilia Leal } \\ \text { University of Illinois } & \text { University of Illinois } & \text { University of Illinois } & \text { University of Illinois } \\ \text { at Urbana-Champaign } & \text { at Urbana-Champaign } & \text { at Urbana-Champaign } & \text { at Urbana-Champaign } \\ \text { kkang14@illinois.edu } & \text { mgoodman@illinois.edu jakrogst@illinois.edu } & \text { cecilial@illinois.edu }\end{array}$

\author{
Dalla R Trinkle \\ University of Illinois \\ at Urbana-Champaign \\ dtrinkle@illinois.edu
}

\author{
Pinshane Y Huang \\ University of Illinois \\ at Urbana-Champaign \\ pyhuang@illinois.edu
}

\author{
André Schleife \\ University of Illinois \\ at Urbana-Champaign \\ schleife@illinois.edu
}

We have incorporated computational modules into the undergraduate courses of Materials Science and Engineering in University of Illinois at Urbana-Champaign. Students have experienced with computational skills in molecular dynamics, density functional theory, and thermodynamics. In Fall of 2019, visualization components were added to a freshman course, to assist in understanding materials' three-dimensional structures early on. As the use of materials databases has surged, in Fall of 2020 we also integrated the current module with a materials database to make students more comfortable with it. Students are supposed to find materials in the database and download corresponding crystallographic files, which are then interfaced with the visualization program, to analyze their structure and symmetry. Students are also asked to compare the visualization result with experimental results. To evaluate the outcome of these modifications, a detailed survey was executed, and the answers about utilization and satisfaction were collected. After the update on exercises, the attitude of students to computation experience in this class becomes more positive. The majority of students felt comfortable to use the materials database and have willing to use it in the future courses. Student responses will be used to improve the current implementation of the module. 